\title{
Häckningsframgång hos svarthakedopping Podiceps auritus i Västergötland, sydvästra Sverige
}

\author{
Breeding success in Horned Grebe Podiceps auritus in Västergötland, Southwest \\ Sweden
}

THOMAS KARLSSON

\begin{abstract}
Breeding success of the Horned Grebe Podiceps auritus was controlled during 2005-2011 at 41 breeding sites with a median size of 1.07 ha in Västergötland, southwest Sweden. The number of pairs varied between 39 and 51. The production of young was 0.99 per pair and year. In natural water bodies there were 1.23 chicks per pair and year and $68 \%$ of the breeding pairs got one or more chicks per pair and year. In artificial water bodies the corresponding values were 0.92 and $50 \%$. Breeding sites with one pair produced 1.29 young per pair and year, compared to 0.85 young where two or more

pairs were breeding at the same site, a difference of 0.44 young. There was a similar difference $(0.48$ young) for artificial water bodies holding one pair or more than one pair, respectively, in contrast to natural water bodies for which the difference was small. There were no indications that the number of young was higher at breeding sites with Mew Gulls or Black-Headed Gulls.

Thomas Karlsson, Brunnsbacken 7, 47550 Hälsö. tk.golfbird@telia.com
\end{abstract}

Received 28 August 2014, Accepted 23 October 2015, Editor: S. Svensson

\section{Inledning}

Häckningsframgång hos den nordvästeuropeiska populationen av svarthakedopping Podiceps auritus har undersökts i olika områden i Norge, Finland och Island (Fjeldså 1973a, Ulfvens 1988), Skottland (Ewing m.fl. 2013, Summers m.fl. 2009) och i Nordamerika (bl.a. Fournier \& Hines 1999, Ferguson \& Sealy 1983). Från Sverige finns uppgifter från slutet av 1960-talet avseende den Gotländska populationen (Högström 1970). De uppgifter om antalet ungar per par, som anges i Tabell 1 spänner från 0,31 till 4,1 ungar per par i olika områden. Häckningsframgång kan t.ex. bero på häckningslokalens kvalitet i form av yta, djup, vegetation, andra häckande sjöfåglar, födotillgång och hot $\mathrm{i}$ olika former (Fjeldså 1973b, Ulfvens 1988, Högström 1970, Fournier \& Hines 1999, Summers m.fl. 2009). Predation av fisk och fåglar påverkar svarthakens förekomst och troligen också häckningsframgång (Ferguson \& Sealy 1983, Summers m.fl. 2009, 2011). Exempelvis föreslår Summers m.fl. (2009) att vid vatten i Skottland med häckande svarthakedoppingar bör inte gädda Esox lucius inplanteras då denna fiskart utgör ett uttalat hot mot svarthaken.
Det finns en uppfattning att svarthakedoppingen gynnas av att häcka ihop med skrattmåskolonier (Tjernberg 2011, Högström 1970). Med skrattmåsens Croicocephalus ridibundus tillbakagång under 1980- och 1990-talen skulle även detta ha missgynnat svarthakedoppingen (Tjernberg 2011). Detta är ett samband som Svensson m.fl. (1999) också berör men inte finner sannolikt. Samma källa nämner att svarthaken ofta häckar i dammar, artificiella vatten, sjöar och naturliga vatten, utan närvaro av skrattmås. En viktigare orsak till en begränsad häckningsförekomst av svarthakedopping torde snarare vara födokonkurrens från fisk (Svensson m.fl. 1999, Tjernberg 2011). Även Högström (1970) finner i sin undersökning att svarthaken inte gynnas av att samhäcka med skrattmås.

Däremot har jag haft svårigheter att finna uppgifter om häckningsutfallet vid häckningslokaler som hyser olika antal par svarthakedopping. I samband med besök vid svarthakelokaler har jag tyckt mig se att ensamma par haft fler ungar. Därför har jag undersökt häckningsframgången hos svarthakedopping i en population i Västergötland i sydvästra Sverige under åren 2005-2011. Huvudsyftet har alltså varit att undersöka om det finns någon skill- 
Tabell 1. Medelantalet ungar per par i förhållande till ungarnas ålder, andel lyckade häckningar och häckningslokalernas arealer.

Mean number of chicks per pair in relation to age of chicks, proportion of successful breeding attempts and area of breeding sites.

\begin{tabular}{|c|c|c|c|c|c|c|c|c|}
\hline \multirow{2}{*}{$\begin{array}{l}\text { Område } \\
\text { Area }\end{array}$} & \multirow{2}{*}{$\begin{array}{l}\text { Referens } \\
\text { Reference }\end{array}$} & \multicolumn{5}{|c|}{ Ålder i dagar Age in days } & \multirow{2}{*}{$\begin{array}{l}\text { Lyckade } \\
\text { häckningar } \\
\text { Successful } \\
\text { breedings }\end{array}$} & \multirow[t]{2}{*}{ Area ha } \\
\hline & & 10 & 20 & $23-28$ & $45-50$ & $\begin{array}{l}\text { Okänd } \\
\text { Unknown }\end{array}$ & & \\
\hline $\begin{array}{l}\text { Bodö-trakten, } \\
\text { Norge }\end{array}$ & Fjeldså 1973a & $2,24^{b}$ & $1,90^{\mathrm{b}}$ & & $1,88^{\mathrm{b}}$ & & $84 \%$ & 0,54 ha \\
\hline Myvatn, Island & Fjeldså $1973^{\mathrm{a}}$ & & & & & $1,06^{\mathrm{a}}-1,9^{\mathrm{b}}$ & $75,5 \%$ & \\
\hline $\begin{array}{l}\text { Yellowknife, } \\
\text { Kanada }\end{array}$ & $\begin{array}{l}\text { Fournier \& } \\
\text { Hines } 1999\end{array}$ & & $2,3^{\mathrm{a}}$ & & & & $60 \%$ & $\begin{array}{l}0,4 \text { ha } \\
1,0 \text { ha } \\
\text { Sjö } \\
\text { Lakes }\end{array}$ \\
\hline $\begin{array}{l}\text { Minnesota, } \\
\text { Kanada }\end{array}$ & $\begin{array}{l}\text { Ferguson \& } \\
\text { Sealy } 1983 \\
\end{array}$ & & & & & $4,1^{\mathrm{c}}$ & & 1,2 ha \\
\hline Skottland & $\begin{array}{l}\text { Ewing m.fl. } \\
2013\end{array}$ & & & & & $\begin{array}{l}0,58 \\
(0,2-1,06)^{\mathrm{a}}\end{array}$ & & \\
\hline Skottland & $\begin{array}{l}\text { Summers m.fl. } \\
2009\end{array}$ & & & & & $\begin{array}{l}0,59^{\mathrm{a}} ; 1992 \\
0.31^{\mathrm{a}} ; 1993 \\
\end{array}$ & $\begin{array}{l}53 \% \\
65 \% \\
\end{array}$ & $\begin{array}{l}5,5 \text { ha } \\
\text { median }\end{array}$ \\
\hline Finland sjöar lakes; & Ulvens 1988 & & & & $\begin{array}{l}1,52 \\
(1,33)\end{array}$ & & & \\
\hline $\begin{array}{l}\text { Finland brackvatten } \\
\text { coastal water }\end{array}$ & Ulvens 1988 & & & & 3,1 & & $59,2 \%$ & \\
\hline Sjöar, Finland Lakes & Uusitalo $1976^{\mathrm{c}}$ & & & & & 1,33 & & \\
\hline $\begin{array}{l}\text { Gotland } \\
\text { Ungar/lokal och år }\end{array}$ & $\begin{array}{l}\text { Högström } \\
1970\end{array}$ & & & & & $\begin{array}{l}0,5 \text { agmyr } \\
\text { Claudium mire } \\
1,1 \text { sjö Lake } \\
2,7 \text { dammar } \\
\text { Ponds }\end{array}$ & $58 \%$ & $\begin{array}{l}14 \text { ha Alla } \\
\text { lokaler } \\
\text { All sites }\end{array}$ \\
\hline
\end{tabular}

$\mathrm{a}=$ Per totalt antal par. $\mathrm{b}=$ per lyckad häckning. $\mathrm{c}=$ Uuisitalo (1976) återges hos Ulfvens (1988).

$a=$ Per total number of pairs. $b=$ per successful pair, $c=$ Uuisitalo (1976) in Ulfvens (1988).

nad mellan häckningslokaler med ensamma par kontra häckningslokaler med samhäckande par. Vidare undersöktes om knipa Bucephala clangula, skrattmås och fiskmås Larus canus påverkade häckningsresultatet.

\section{Metodik}

Förekomsten av svarthakedopping vid potientella häckningslokaler noterades vid minst två besök, det första under andra halvan av maj och därefter minst ett besök från första veckan i juli månad eller senare under sommaren tills häckningsutfallet kunde fastställas, i enlighet med vad som beskrivs av Regnell (1981). En sammanvägd bedömning av antalet par gjordes på basis av observationer vid de olika besökstillfällena. Antalet ungar noterades endast en gång, oavsett deras ålder, och häcknings- utfallet registrerades som antalet ungar per par. Observation av ett par med minst en unge klassificerades som en lyckad häckning.

En häckningslokal definierades som ett vatten med en sammanhängande vattenyta utan hinder. Fanns det tydligt avgränsade delar av samma vatten med vallar, staket eller annat som gjorde att doppingarna inte kunde simma fritt mellan olika delar, utgjorde dessa separata häckningslokaler. Häckningsutfallet har jämförts mellan naturligt skapade vatten (sjöar) och konstgjorda vatten (dammar) i enlighet med Norevik (2014). Som ett mått på det genomsnittliga häckningsutfallet på de olika lokalerna beräknades medelantalet ungar per par och år för var och en av dem (Primärdata i Appendix 1 och 2).

Flytbladvegetationens utbredning skattades i förhållande till vattenytan genom enkel skattning 
till närmaste $10 \%$, där $1 \%$ visade att det åtminstone kunde ses några blad och $100 \%$ att hela sjöytan var täckt.

Fynd av knipa, skrattmås och fiskmås, både häckande och icke häckande, registrerades på för varje häckningsplats. Uppgifter om fiskfaunan har erhållits genom att fråga markägare, kommunekolog eller annan sakkunnig som kunde tänkas ha någon kunskap om förhållandena i häckningslokalen.

Materialet har bearbetats och sammanställts på årsbasis varefter skillnader analyserats med Wilcoxons rangsummetest, Mann-Whitney's test och $\mathrm{Chi}^{2}$-test.

\section{Undersökningsområde}

Svarthakedoppingens förekomst i Västergötland tycks vara av relativt sent datum där den första kända häckningen i området ägde rum 1931 i Hornborgasjön, medan de centralare delarna av det västgötska utbredningsområdet kanske inte besattes förrän under 1950-talet. Beståndet av svarthakedopping i Västergötland tycks ha varit relativt stabilt och uppskattades under perioden 1970 och fram till riksinventeringen 1996 ha legat på 60-70 par (Karlsson \& Brander 1997, Douhan 1998). Även vid riksinventeringen 2011 fann man likartade siffror (Norevik 2014). Merparten av populationen återfinns inom den skaraborgska delen av Västergötland inom kommunerna Falköping, Skövde och Tidaholm.

Årligen har 26-31 lokaler besökts och totalt har undersökningen omfattat 41 lokaler. De flesta lokalerna utgjordes av småvatten med en medianstorlek på 1,07 ha; 17 av dem mindre än 1 ha och nio var större än 2 ha. Av lokalerna var 31 stycken konstgjorda vatten och resten, 10 stycken, var naturliga vatten, i enlighet med definition av Norevik (2014). Fördelningen framgår av Appendix 1. Hälften av häckningslokalerna omgavs av åker eller annan öppen mark, $25 \%$ av lövskog och resten av barrskog som dominerande inslag runt häckningslokalen. Lokalerna ute i åkermarken var i samtliga fall konstgjorda vatten.

Flytbladsvegetationen har i första hand bestått av näckros och gäddnate Potamogeton natans.

Fiskfaunan är dåligt känd. Ett försök att reda ut förekomsten av gädda i svarthakelokalerna, eftersom gäddan bedöms ha stor inverkan på svarthakedopping (Summers m.fl. 2009), har gjorts. Endast från 21 lokaler har det hittills gått att få säkra uppgifter. Vid en lokal har gädda noterats och vid övriga 20 lokaler saknas den (Appendix 1).

Utöver svarthakedopping noterades förekomst av fiskmås, skrattmås och knipa. Knipa häckade vid alla lokaler utom två mycket små vatten på 0,08 respektive 0,13 ha. Fiskmås fanns vid 26 lokaler, oftast med endast ett par. Fem häckningsvatten hyste mindre skrattmåskolonier, men endast 2 av dem under samtliga inventerade år.

\section{Resultat}

\section{Antal par}

Antalet par i undersökningen varierade mellan 39 och 51 per år (primärdata Appendix 2), vilket betyder att av de 40-80 par som bedömts häcka i Västergötland (Ottosson m.fl. 2012) har cirka 70\% ingått i undersökningen. Fördelningen av antalet par med avseende på konstgjorda och naturliga vatten var i medeltal 34,7 respektive 10,0 par per år $(n=7)$ och antydde inte preferens för någon av de två typerna av vatten $\left(\mathrm{Chi}^{2}=3,49\right.$, df 6 , ej sign $)$.

\section{Ungproduktion}

Ungproduktionen var i medeltal över åren 0,99 ungar per par och år. Antalet ungar i kullarna varierade mellan ett och fem. Kullar med en unge dominerade, $44 \%$, medan bara $6 \%$ av kullarna bestod av 4-5 ungar (Figur 1).

Ungproduktionen var bättre i naturliga vatten med i medeltal 1,23 ungar per par och år än $\mathrm{i}$ konstgjorda vatten med 0,92 ungar per par och år (Tabell 2). I medeltal lyckades 54\% av häckningarna, men i konstgjorda vatten endast $50 \%$ jämfört med 68\% för naturliga vatten (Tabell 3). Dessutom visade det sig att ensamhäckande par producerade 1,29 ungar per par och år medan par med grannar i samma vatten fick 0,85 ungar per par, en skillnad på 0.44 ungar (Tabell 4). Procentandelen lyckade

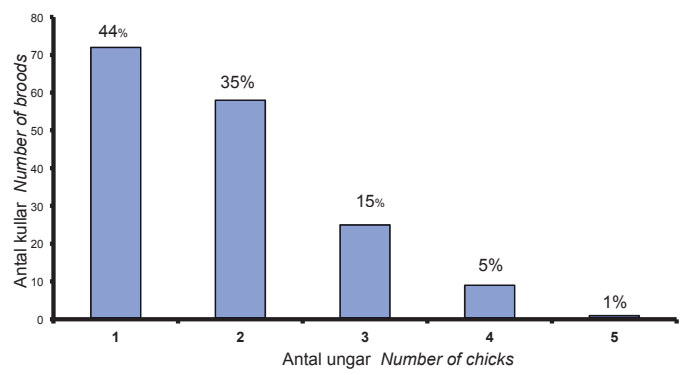

Figur 1. Fördelningen av ungkullar av olika storlek, totalt under hela undersökningsperioden (165 kullar).

The distribution of broods of different size, total during the whole study period (165 broods). 
Tabell 2. Medelantal ungar per par.

Mean number of young per pair.

\begin{tabular}{|c|c|c|c|c|c|c|}
\hline \multirow[b]{2}{*}{$\begin{array}{l}\text { Ar } \\
\text { Year }\end{array}$} & \multicolumn{2}{|l|}{$\begin{array}{l}\text { Hela materialet } \\
\text { Whole data set }\end{array}$} & \multicolumn{2}{|c|}{$\begin{array}{l}\text { Naturliga vatten } \\
\text { Natural water bodies }\end{array}$} & \multicolumn{2}{|c|}{$\begin{array}{l}\text { Konstgjorda vatten } \\
\text { Artificial water bodies }\end{array}$} \\
\hline & $\begin{array}{l}\text { Ungar per par } \\
\text { Young per } \\
\text { pair }\end{array}$ & $\begin{array}{l}\text { Antal par } \\
\text { Number of } \\
\text { pairs }\end{array}$ & $\begin{array}{c}\text { Ungar per par } \\
\text { Young per } \\
\text { pair }\end{array}$ & $\begin{array}{c}\text { Antal par } \\
\text { Number of } \\
\text { pairs }\end{array}$ & $\begin{array}{l}\text { Ungar per par } \\
\text { Young per } \\
\text { pairs }\end{array}$ & $\begin{array}{c}\text { Antal par } \\
\text { Number of } \\
\text { pairs }\end{array}$ \\
\hline 2005 & 1,69 & 39 & 1,75 & 8 & 1,68 & 31 \\
\hline 2006 & 1,05 & 39 & 1,29 & 7 & 1,00 & 32 \\
\hline 2007 & 0,69 & 48 & 1,00 & 12 & 0,58 & 36 \\
\hline 2008 & 0,80 & 45 & 1,00 & 12 & 0,73 & 33 \\
\hline 2009 & 1,05 & 41 & 1,50 & 10 & 0,90 & 31 \\
\hline 2010 & 0,80 & 51 & 1,25 & 8 & 0,72 & 43 \\
\hline 2011 & 0,88 & 50 & 0,85 & 13 & 0,85 & 37 \\
\hline $\begin{array}{l}\text { Medelvärde } \\
\text { Mean }\end{array}$ & 0,99 & & 1,23 & & 0,92 & \\
\hline
\end{tabular}

Anm.: Parvisa jämförelser mellan naturliga och konstgjorda häckningsvatten antyder att ungproduktionen har varit högre $\mathrm{i}$ naturliga vatten (Wilcoxons rangsummetest avseende matchade par; $\mathrm{p}=0,03$; tvåsidigt).

Note: Pair-wise comparisons between "lakes" and "ponds" indicate a higher production of young at natural water bodies (Wilcoxons signed rank test; $p=0.03 ;$ two-tailed).

Tabell 3. Procentandel lyckade häckningar. $\mathrm{N}=$ number of pairs.

Percentage of successful breedings. $N=$ antal par.

\begin{tabular}{llllllc}
\hline & $\begin{array}{c}\text { Hela materialet } \\
\text { Whole data set }\end{array}$ & $\begin{array}{c}\text { Naturliga vatten } \\
\text { Lakes, natural } \\
\text { waterbodies }\end{array}$ & $\begin{array}{c}\text { Konstgjorda vatten } \\
\text { Ponds, artifical } \\
\text { waterbodies }\end{array}$ \\
\hline År Year & $\%$ & $\mathrm{~N}$ & $\%$ & $\mathrm{~N}$ & $\%$ & $\mathrm{~N}$ \\
\hline 2005 & 77 & 39 & 88 & 8 & 74 & 31 \\
2006 & 56 & 39 & 71 & 7 & 53 & 32 \\
2007 & 50 & 48 & 58 & 12 & 47 & 36 \\
2008 & 44 & 45 & 58 & 12 & 39 & 33 \\
2009 & 59 & 41 & 80 & 10 & 52 & 31 \\
2010 & 41 & 51 & 75 & 8 & 35 & 43 \\
2011 & 48 & 50 & 46 & 13 & 49 & 37 \\
\hline Medelvärde Mean & 54 & & 68 & & 50 & \\
\hline
\end{tabular}

Anm.: Parvisa jämförelser mellan konstgjorda och naturliga vatten antyder att andelen lyckade häckningar har varit något högre i naturliga vatten (Wilcoxons rangsummetest avseende matchade par; $\mathrm{p}=0,03$; tvåsidigt).

Note: Pair-wise comparisons between "lakes" and "ponds" indicates a higher precentage of successful breeding attempts at natural water bodies (Wilcoxon signed rank test; $p=0.03$; two-tailed).

häckningar var $65 \%$ respektive $49 \%$ (Tabell 5). Skillnaden kvarstod även när man enbart jämförde konstgjorda vatten, 1,25 ungar per par och år för ensamhäckande respektive 0,77 ungar per par och år för par med grannar i samma vatten, en skillnad på 0,48 ungar (Wilcoxons rangsummetest för matchade par, $p=0,02$, tvåsidigt). En motsvarande tendens fanns även för naturliga vatten men den kunde inte verifieras med statistisk signifikans, 1,35 respektive 1,08 ungar per och år, skillnad 0,27 ungar (Wilcoxons rangsummetest för matchade par, $\mathrm{p}=0,30$, tvåsidigt).

Det finns ingen indikation på tidstrend under den aktuella sjuårsperioden (Spearmans rangkorrelatonskoefficient, $r_{s}=-0,29$, ej sign.). 
Tabell 4. Medelantal ungar per par, relaterat till om det funnits ett eller flera par på lokalen. $\mathrm{N}=$ antal par. Mean number of chicks per pair, related to the presence of one or more pair at the site. $N=$ number of pairs.

\begin{tabular}{lllll}
\hline \multirow{2}{*}{ Ar Year } & \multicolumn{2}{c}{ 1 par pair } & \multicolumn{2}{c}{$>1$ par pair } \\
& $\begin{array}{c}\text { Medel } \\
\text { Mean }\end{array}$ & $\mathrm{N}$ & $\begin{array}{c}\text { Medel } \\
\text { Mean }\end{array}$ & $\mathrm{N}$ \\
\hline 2005 & 2,13 & 16 & 1,39 & 23 \\
2006 & 1,18 & 17 & 0,95 & 22 \\
2007 & 0,83 & 18 & 0,60 & 30 \\
2008 & 1,13 & 16 & 0,62 & 29 \\
2009 & 1,31 & 13 & 0,93 & 28 \\
2010 & 1,00 & 11 & 0,75 & 40 \\
2011 & 1,45 & 13 & 0,77 & 37 \\
\hline Medel Mean & 1,29 & & 0,85 & \\
\hline
\end{tabular}

Anm.: Parvisa jämförelser per år antyder att ungproduktionen varit högre för ensamhäckande par (Wilcoxons rangsummetest avseende matchade par, $\mathrm{p}=0,02$, tvåsidigt).

Note: Pair-wise comparisons indicate a higher production of chicks among pairs breeding alone (Wilcoxon signed rank test; $p=0.02$; two-tailed).

\section{Häckningsframgång i förhållande till måsförekomst}

Det fanns ingen indikation på skillnader i häckningsutfallet med avseende på förekomsten av häckande måsar. Medelantalet ungar per par, år och lokal var 1,05 för 26 lokaler med fiskmås och 1,26 för 15 lokaler utan häckande fiskmås (Mann-Whitney U-test, $p=0,88$, tvåsidigt). Inte heller för skrattmåsen fanns det någon skillnad; häckningsutfallet var i medeltal 1,21 ungar per par, år och lokal vid närvaro av häckande skrattmås jämfört med 1,14 utan närvaro av skrattmås (Tabell 6).

\section{Inverkan av flytbladvegetationens utbredning}

Skattningar av flytbladsvegetationens utbredning gjordes på 36 lokaler med en genomsnittlig utbredning på $30 \%$. Något samband med medelantalet ungar per par, år och lokal fanns inte (Sperman $r_{s}=0,07, n=36$ lokaler, ej sign., primärdata i Appendix 1).

\section{Förekomst av knipa på häckningslokalen}

Knipa fanns på alla häckningslokaler utom två mycket små lokaler om 0,08 respektive 0,13 ha. På tre lokaler förekom fler knipor än på övriga och där man ofta kunde räkna $\geq 20$ översomrande exemplar. Det fanns en numerärt stor skillnad i ungproduk-
Tabell 5. Procentandelen lyckade häckningar, relaterat till om det funnits ett eller flera par på lokalen. $\mathrm{N}$ $=$ antal par.

Percentage of successful breedings, related to the presence of one or more pair at the site. $N=$ number of pairs.

\begin{tabular}{lllll}
\hline & \multicolumn{2}{c}{ 1 par pair } & \multicolumn{2}{c}{$>$ 1 par pair } \\
\hline År Year & $\%$ & $\mathrm{~N}$ & $\%$ & $\mathrm{~N}$ \\
\hline 2005 & 94 & 16 & 65 & 23 \\
2006 & 64 & 17 & 59 & 22 \\
2007 & 67 & 18 & 40 & 30 \\
2008 & 44 & 16 & 45 & 29 \\
2009 & 62 & 13 & 57 & 28 \\
2010 & 46 & 11 & 37 & 40 \\
2011 & 77 & 13 & 38 & 37 \\
\hline Medel Mean & 65 & & 49 & \\
\hline
\end{tabular}

Anm.: Parvisa jämförelser mellan ensamhäckande och samhäckande par indikerar en högre andel ingen signifikant högre andel lyckade häckningar bland ensamhäckare (Wilcoxons rangsummetest avseende matchade par, $\mathrm{p}=0,03$, tvåsidigt).

Note: Pair-wise comparisons between breeding sites with one pair and two or more pairs indicates a higher percentage of succesful breeding attempts by pairs breeding alone (Wilcoxon signed rank test; $p=0.03$; two-tailed).

tionen på lokalerna med många knipor, i medeltal 0,43 ungar per par, år och lokal jämfört med ett genomsnitt på 1,19 för övriga lokaler, men den var inte statistiskt signifikant (Mann-Whitney U-test, $\mathrm{p}=0,12$, tvåsidigt).

\section{Diskussion}

Häckningsvattnets medianstorlek var i Västergötland 1,07 ha och skiljer sig inte påtagligt från andra populationers (Tabell 1). På Gotland var häckningslokalerna i medeltal 14 ha, men där utgjorde konstgjorda vatten, dammar, endast 3 av 16 redovisade häckningslokaler och medelytan var 4 ha med ett häckningsresultatet som var klart bättre (Högström 1970, Tabell 1). Faaborg (1976) konstaterade att i North Dakota, USA, var $60 \%$ häckningsplatserna mindre än 1 ha. Överlag verkar inte storleken på häckningslokalen variera över världen utan ligga i storleksordning 0,1-2 ha, möjligen med undantag av Skottland där medianstorleken var 5,5 ha (Tabell 1).

Storleken på häckningslokaler där samhäckning skedde var hos Fjeldså (1973a) 1,2 ha på häckningsplatser i Norge och i Mannitoba, Kanada, noterade 
Tabell 6. Medelantal ungar per par och häckningslokal, relaterat till förekomsten av häckande skrattmås. $\mathrm{N}=$ antal lokaler.

Mean number of chicks per pair and breeding site, related to the presence of breeding Black-headed Gull. $N=$ number of sites.

\begin{tabular}{lllll}
\hline \multirow{2}{*}{ År Year } & $\begin{array}{c}\text { Med skrattmås } \\
\text { With Black- } \\
\text { headed gull }\end{array}$ & $\begin{array}{c}\text { Utan skrattmås } \\
\text { Without Black- } \\
\text { headed gull }\end{array}$ \\
\cline { 2 - 5 } & $\begin{array}{l}\text { Medel } \\
\text { Mean }\end{array}$ & $\mathrm{N}$ & $\begin{array}{c}\text { Medel } \\
\text { Mean }\end{array}$ & $\mathrm{N}$ \\
\hline 2005 & 1,33 & 4 & 1,98 & 21 \\
2006 & 0,50 & 3 & 1,17 & 24 \\
2007 & 0,25 & 3 & 0,77 & 28 \\
2008 & 3,00 & 1 & 0,86 & 26 \\
2009 & 1,34 & 2 & 1,13 & 23 \\
2010 & 1,34 & 2 & 0,91 & 23 \\
2011 & 0,74 & 2 & 1,12 & 25 \\
\hline Medel Mean & 1,21 & & 1,14 & \\
\hline
\end{tabular}

Anm.: Parvisa jämförelser antyder att inga skillnader (Wilcoxons rangsummetest avseende matchade par, $\mathrm{p}=0,69$, tvåsidigt).

Note: Mean number of chicks per pair and breeding site, related to the presence of breeding Black-headed Gull. Pairwise comparisons (Wilcoxon signed rank test; $p=0.69$; two-tailed).

Ferguson \& Sealy (1983) detta på lokaler $>1,1$ ha. I den här undersökningen fanns det dubbelhäckningar redan vid så små naturliga vatten som 0,35 ha och vid konstgjorda vatten 0,39 ha. Det tycks som om häckningsvatten runt 0,1 ha ger tillräckligt med föda för att kunna föda en kull och här tycks inte den västgötska populationen särskilja sig. Från 0,35 ha och uppåt finns förutsättingar för mer än ett par. Enligt Fournier \& Hines (1999) föredrar svarthakedoppingen konstgjorda före naturliga vatten med 0,81 par per ha respektive 0,14 par per ha. I Västergötland noterade jag i konstgjorda vatten 1 $\mathrm{par} /$ ha och $0,27 \mathrm{par} /$ ha i naturliga häckningsvatten. Man kan också notera att det är stor skillnad mellan antalet ungar hos samhäckande par i konstgjorda och naturliga vatten där antalet ungar i medeltal är $40 \%$ högre i naturliga vatten än i konstgjorda.

I den här undersökningen lyckades $54 \%$ av paren med att åtminstone få en unge (Tabell 3), men över de 7 åren varierade andelen lyckade par mellan 41\% och $77 \%$. Andelen lyckade häckningar är i nivå med eller något lägre jämfört med andra undersökningar och med reservation för att jämförelser inte är helt lätta att göra på grund av metodskillnader (Tabell 1). I Skottland noterade man en låg ung- produktion på 0,2-1,06 ungar med ett medelvärde på 0,58 ungar per par (Ewing m.fl. 2013, Summers m.fl. 2009, Tabell 1). Den genomsnittliga ungproduktionen över åren i den här undersökningen var 0,99 ungar per par och år (Tabell 2), vilket står sig ganska gott om man jämför med andra populationer. I jämförelse med den skotska population, som dock minskar kraftigt (Brooks m.fl. 2012), verkar populationen i Västergötland vara stabil (Karlsson \& Brander 1997, Norevik 2014).

Ungproduktionen var 52\% högre för par som var ensamma på häckningslokalen jämfört med samhäckande par (Tabell 4) och det fanns även en signifikant skillnad vad gäller procentandelen lyckade häckningar, $65 \%$ för ensamhäckare och $49 \%$ för par med häckande artfränder i samma vatten (Tabell 5). Detta resultat tyder på att förhållanden som påverkar äggkullens storlek och/eller ungarnas överlevnad kan ha varit gynnsammare för ensamhäckande par än för samhäckande par och att konkurrens om t.ex. föda till ungarna kan ha varit en bakomliggande faktor. Av observationer under inventeringen att döma skulle en orsak till detta vara att där två eller fler par samhäckar, har de fullt upp med att bevaka sitt revir så att det inte blir tid över för själva häckningen. Vid observationstillfällena var skärmyttslingar mellan två par en vanlig syn, vilket även Brander \& Karlsson (2006) noterade. Faaborg (1976) förklarar att boplatsvalet kan vara en orsak till lägre antal ungar vid samhäckning då andra paret får bygga sitt bo på en plats mer utsatt för vädret, vilket skulle leda till att framför allt ägg faller i vattnet och inte kläcks. Faaborg (1976) noterade också att i vatten med samhäckande par var de mer aggressiva mot andra svarthakar. Det kan också tänkas att bättre födotillgång under framför allt äggläggningsperioden slutligen ger fler ungar för ensamhäckande par.

När det gäller födokonkurrens noterade jag att knipa fanns på nästan samtliga häckningsplatser med åtminstone en kull och vid några lokaler fanns översomrande knipor. Knipan har liksom svarthakedoppingen en diet dominerad av vattenlevande insekter (t.ex. Bauer m.fl. 1969, Eriksson 1976, Fjeldså 1973b och Ulvens 1988), så födotillgången skulle kunna utgöra en begränsande faktor. Enstaka par av knipa verkar inte ha någon större betydelse medan större mängder översomrande knipor kan tänkas ha inverkan på häckningsutfallet. Utfall mot knipor var också en vanlig syn under inventeringen.

I Artdatabankens artfaktablad (Tjernberg 2011) antyds att ett minskat antal skrattmåskolonier åtminstone lokalt torde ha påverkat förekomsten 
av häckande svarthakedoppingar, men i den här undersökningen upptäcktes ingen skillnad i häckningsutfallet på lokaler med respektive utan häckande skrattmås (Tabell 6). Tjernberg (2011) och Ulfvens (1988) noterar att flertalet häckningar faktiskt inte sker på lokaler med skrattmås, vilket bekräftas här. Snarare är det kanske så att i de fallen arterna samhäckar så har häckningslokalen en struktur som gynnar båda arterna. Man kan också tänka sig att fiskmås skulle kunna ha en liknande skyddande effekt på svarthakedopping, men även här visar undersökningen att det inte finns någon skillnad.

Från Skottland rapporteras att spigg Gasterosteidae sp. och elritsa Phoxinus phoxinus utgör en viktig födoresurs. De båda fiskarterna utgör också en viktig föda för gädda (Summers m.fl. 2009 och 2011). Summers m.fl. (2009) fann emellertid att ungförlusterna var 5,9 gånger högre $\mathrm{i}$ vatten där gädda fanns än i andra vatten. I den här undersökningen har jag försökt få fram uppgifter om gädda. Trovärdiga uppgifter från uppgiftslämnare med kännedom häckningslokalerna har bara erhållits för 21 lokaler (Appendix 1), varav bara en med säkerhet hyst gädda. Detta kan vara en indikation på att svarthakedoppingen i första hand väljer gäddfria vatten för sin häckning, men för en säkrare bedömning behövs kompletterande undersökningar av fiskförekomsten i fler småvatten i undersökningsområdet. I varken den här undersökningen eller den som utfördes av Brander \& Karlsson (2006) gjordes iakttagelser av födoöverlämning som bestod av fisk.

\section{Tack}

Mitt särskilda tack till Mats Eriksson som på ett högst väsentligt sätt har bidragit med mycket värdefulla förbättringar av manus och statistiska beräkningar. $\mathrm{H}$. Lerner granskade ett tidigare manusförslag. En anonym referent tackas likaså för förslag till förbättringar av manus. Herrarna L. Friggeråker, J. Grahn, M. Hallgren och C.-O. Persson tackas för att de delgivit mig uppgifter om häckningslokaler. Ett viktigt logistikstöd har erhållits från S. Ottzén, L. \& G. Palm Karlsson samt M. \& S. Koszinowski. Ett ekonomiskt stöd utgick 1995 till undersökningen från Elis Wides fond (Sveriges Ornitologiska Förening) och som äntligen kom till användning.

\section{Referenser}

Bauer, K.M. \& Glutz von Blotzheim, U.N. 1969. Handbuch der Vögel Mitteleuropas. Band 3. Akademische Verlagsgesellschaft. Wiesbaden.

Brander, A., \& Karlsson, T. 2006. Matning av ungar hos svarthakedopping Podiceps auritus. Ornis Svecica 16: 177-182.

Brooks, S.J., Jones, V.J., Telford, R.J., Appleby, P.G., Watson, E., McGowan, S. \& Benn, S. 2012. Population trends in the Slavonian grebe Podiceps auritus (L.) and Chironomidae (Diptera) at a Scottish loch. J. Paleolimnol. 47: 631-644.

Douhan, B. 1998. Svarthakedoppingen - en fågel på tillbakagång i Sverige. Vår Fågelvärld 57: 7-22.

Eriksson, M.O.G. 1976. Food and feeding habits of downy Goldeneye Bucephala clangula (L.) ducklings. Ornis Scandinavica 7: 159-169.

Ewing, S.R., Benn, S., Cowie, N., Wilson, L. \& Wilson, J.D. 2013. Effects of weather variation on a declining population of Slavonian Grebes Podiceps auritus. J. Ornithol. 154: 955-1006.

Faaborg, J. 1976. Habitat selection and territorial behaviour of the small grebes of North Dakota. The Wilson Bulletin 88: 390-399.

Ferguson, R.S. \& Sealy, S.G. 1983. Breeding ecology of the Horned Grebe, Podiceps auritus, in Southwestern Manitoba. Canadian Field-Naturalist 97(4): 401-408.

Fjeldså, J. 1973a. Territory and the regulation of population density and recruitment in the horned grebe Podiceps auritus arcticus Boje, 1822. Vidensk. Meddr. Dansk naturh. Foren. 136: 117-189.

Fjeldså, J. 1973b. Feeding and habitat selection of the horned grebe Podiceps auritus (Aves), in the breeding season. Vidensk. Meddr. Dansk naturh. Foren. 136: 57-95.

Fournier, M.A. \& Hines, J.E. 1999. Breeding ecology of the Horned Grebe Podiceps auritus in subartic wetlands. Canadian Wildlife Service Occasional Paper Nr 99: 1-32. ISBN 0576-6370.

Högström, S. 1970. Svarthakedoppingen Podiceps auritus på Gotland. Vår Fågelvärld 29: 60-66.

Karlsson, T. \& Brander, A.-K. 1997. Svarthakedoppingen i Skaraborg. Grus 23: 33-49.

Norevik, G. 2014. Horned Grebe Podiceps auritus and rednecked Grebe Podiceps grisegena in Sweden 2011 - results from a national survey. Ornis Svecica 24: 81-98.

Ottosson, U., Ottvall, R., Elmberg, J., Green, M., Gustafsson, R., Haas, F., Holmqvist, N., Lindström, Å., Nilsson, L., Svensson, M., Svensson, S. \& Tjernberg, M. 2012. Fåglarna i Sverige - antal och förekomst. SOF, Halmstad.

Regnell, S. 1981. Häckningsbeståndet av svarthakedopping Podiceps auritus i Sverige. Vår Fågelvärld 40: 13-22.

Summers, R. W., Mavor, R. \& Hancock, M. H. 2009. Correlates of Breeding Success of Horned Grebes in Scotland. Waterbirds 32(2): 265-275.

Summers, R. W., Roddy, A. M., Hogg, S. \& Harriman, R. 2011. Lake characteristics and their selection by breeding Slavonian Grebes Podiceps auritus in Scotland. Bird Study 58: 349-356.

Svensson, S., Svensson, M. \& Tjernberg, M. 1999. Svensk Fågelatlas. Vår Fågelvärld, supplement 31, Stockholm.

Tjernberg, M. 2011. Svartakedopping. http://www.artfakta. se/Artfaktablad/Podiceps_Auritus_100113.pdf 
Ulfvens, J. 1988. Comparative breeding ecology of the Horned Grebe Podiceps auritus and the Great Crested Grebe Podiceps cristatus: archipelago versus lake habitats. Acta Zool. Fenn. 183: 1-75.

\section{Summary}

An investigation of breeding success of the Horned Grebe Podiceps autritus was done during 20052011 at 41 breeding sites in Västergötland, southwest Sweden. Breeding success in the Northwest European population of the Horned Grebe have been investigated in Finland, Norway, and Iceland (Ulfvens 1988, Fjeldså 1973a), Scotland (Ewing et al. 2013, Summer et al. 2009) and in North America (Fournier \& Hines 1999, Ferguson \& Sealy 1983). In Sweden the species has been studied during 1960s at Gotland (Högström 1970). The number of chicks per pair in these investigations varied between 0.31 and 4.1 (Table 1). Differences in breeding success depend on the quality of the breeding in terms of where area, depth, vegetation, other seabirds, and the amount of food (Fjeldså 1973b, Ulfvens 1988, Högström 1970, Fournier \& Hines 1999, Summers et al. 2009). Predation by fish and birds is also important (Ferguson \& Sealy 1983, Summers et al. 2009, 2011).

The Horned Grebe has been proposed to be favoured by breeding in colonies of the Blackheaded Gull (Tjernberg 2011, Högström 1970, Svensson et al. 1999, Ulfvens 1988), but none of these authors found it likely. It is more likely that breeding success depend on predation and/or food competetion by fish (Svensson m.fl. 1999, Tjernberg 2011).

The primary aim of this study was to investigate any difference in breeding success between pairs breeding alone or together with other pairs in the same water body, but also if there is any advantage of breeding together with Mew Gull Larus canus or Black-headed Gull Croicocephalus ridibundus.

\section{Methods}

Each breeding sites was checked twice per breeding season, the second half of May and from the first week of July until any chicks could be seen (Regnell 1981). The number of pairs was compiled from these observations. The number of chicks was recorded once. The number of chicks per pair was compared between natural and artificial water bodies, following the terminology by Norevik (2014). The cover of floating vegetation was estimated to nearest $10 \%$ where $1 \%$ showed at least some leaves in the water and $100 \%$ that the whole surface was covered. Observations of Goldeneye Bucephala clangula, Black-headed Gull and Mew Gull were recorded. Information about the fish fauna was obtained by asking landowners, nature conservationists or others, and information was obtained from 21 sites.

In total, 41 breeding sites were investigated and the number of visisted sites varied between 26-31 waters in single years (details in Appendix 1). The fish fauna in general is poorly known but the potentially important predator Pike Esox lucius was known in only one water (details in Appendix 1). Goldeneye was found breeding at all 41 grebe sites except for two very small waters, 0.08 and 0.13 ha.

\section{Results and discussion}

The number of Horned Grebe pairs varied between 39 och 51 (Table 2), which corresponded to approximately $70 \%$ of the population in Västergötland (Ottosson et al. 2012). In artificial waters there were on avererage 34.7 pairs per year and in natural waters 10.0 pairs per year $(n=7)$, with no preference $\left(\mathrm{Chi}^{2}=3.49\right.$; df 6 ; ns). Overall, $54 \%$ of the pairs were successful in rearing at least one chick, in artifical waters $50 \%$ och in natural waters $68 \%$ (Table 3).

The number of chicks per brood varied between 1 and 5. Broods with one chick were predominant, $44 \%$, while broods with 4 and 5 young comprised $6 \%$ of the broods (Figure 1). Overall there were 0.99 chicks per pair and year; in natural waters 1.23 compared to 0.92 in artificial waters (Table 2). Waters with one pair produced 1.29 chicks per pair and year compared to waters with two or more pairs which produced 0.85 young per pair and year (Table 4). This difference was also true for artificial waters, where one pair got 1.25 young per pair and year and two or more pairs 0.77 young per pair and year (Wilcoxons signed rank test for matched pairs; $p=0.02$; two-tailed). In natural water bodies the difference was 1.35 and 1.08 respectively and not significant (Wilcoxons signed rank test for matched pairs, $\mathrm{p}=0.30$, two-tailed). $54 \%$ of the pairs were successful in getting at least one chick (Table 3), but it varied between $41 \%$ and $77 \%$ and thus somewhat lower than other study areas (Table 1).

At sites with breeding Mew Gulls there was no difference in breeding success; 1.05 chicks per pair, year and site for 26 sites with breeding Mew Gulls, compared to 1.26 for 15 sites without breeding gulls (Mann-Whitney U-test, $\mathrm{p}=0.88$, two- 
tailed). Nor was there any difference between sites with and without breeding Black-headed Gull, 1.21 and 1.14 chicks per pair year and sites, respectively (Table 6).

The coverage of floating vegetation was estimated at 36 breeding sites. There was no correlation between coverage and the mean number of chicks per pair, year and site (Sperman $r_{s}=0.07, n=36$ sites, n.s.).

The median area of the breeding water in Västergötland was 1.07 ha, which is in the range of $0.1-2$ ha found in other studies, except for a study in Scotland where the median size was 5.5 ha (Table 1). In my study most of the waters were ponds, in Scotland lakes. Breeding of two ore more pairs at the same water body was recorded at ponds from a surface area of 1.2 ha in Norway (Fjeldså 1973a), and in Mannitoba, Canada, Ferguson \& Sealy (1983) noticed that two pairs bred at water bodies of 1.1 ha. In this study, breeding of two or more pairs was recorded at artificial water bodies from 0.35 ha and at natural water bodies from 0.39 ha.

Over the years the production was 0.99 young per pair and year (Table 2), which is good comparing to the population i Scotland (Ewing m.fl. 2013, Summers m.fl. 2009, Table 1), but more difficult to compare with other investigations due to different methods. The Scottish population has been decreasing for a number of years while the population in Västergötland is stable. The number of chicks per pair where the pair was alone at the breeding site was $52 \%$ higher compared to breeding sites with several pairs (Table 4). There was also a significant higher perecentage of successful breeding attempts among pairs breeding alone compared to pairs with conspecific neighbours in the same water, $65 \%$ and $49 \%$ respectively (Table 5 ). This result indicates more favourable conditions for pairs breeding alone, perhaps with reference to any competition for e.g. food for the chicks.

Breeding together with Goldeneye is common and there was at least one pair on every breeding site, except for two very small sites. At three sites more than 20 Goldeneyes stayed more regularly during summer, but there was no significant difference breeding success at these sites compated to the remaining ones.

With reference to fish, Pike was reported to occur at only one of 21 sites with available information. Whether this result reflects that Horned Grebe avoid breeding at water bodies with Pike in the study are remains to be clarified. 


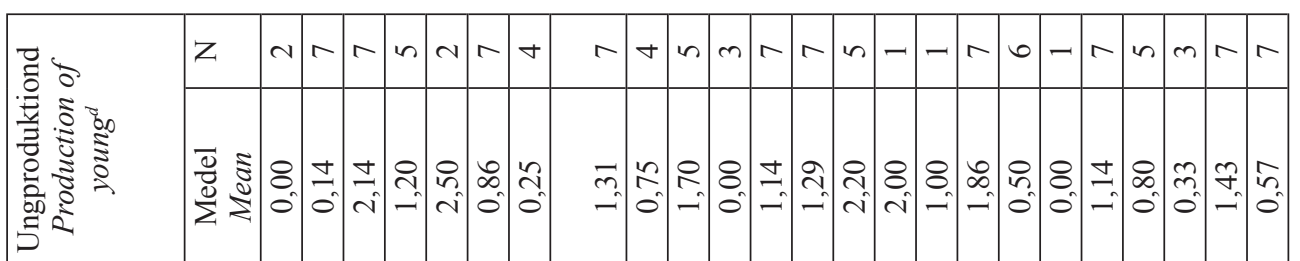

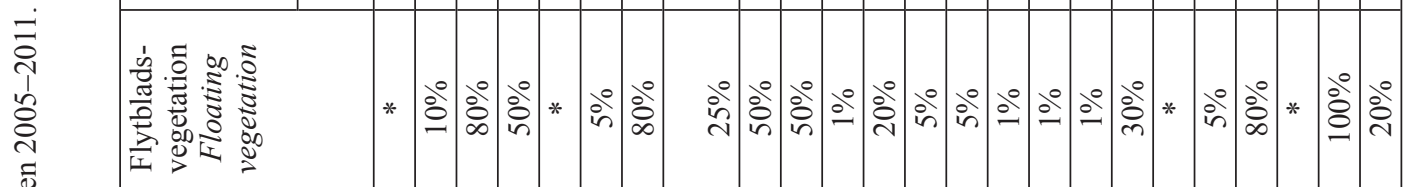

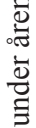

\begin{tabular}{|c|c|c|c|c|c|c|c|c|c|c|c|c|c|c|c|c|c|c|c|c|}
\hline & $\frac{\pi}{\frac{\pi}{0}}$ & 0 & * & * & -0 & o & 0 & 0 & * & * & * 0 & $*$ & * & * & 10 & 0 & * & 6 & $*$ & 0 \\
\hline & 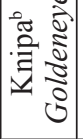 & 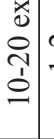 & $\stackrel{\sim}{\perp}$. & -1 & $\stackrel{I}{\perp}-$ & $-I$ & $\stackrel{I}{-}$ & $\stackrel{I}{I}$ & - & $\stackrel{I}{I}$ & 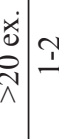 & - & - & - & $-1-$ & $\neg$ & -- & -- & - & - \\
\hline 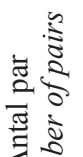 & 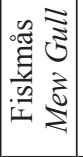 & $\stackrel{1}{-}$ & - & & & & -1 & - & $\hat{r}$ & $\stackrel{\imath}{\imath}$ & - & - & -1 & -1 & $-1-$ & & & -- & $\underline{f}$ & -1 \\
\hline $\bar{z}$ & 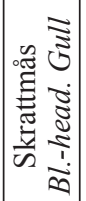 & & & & & & & 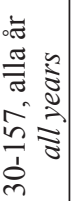 & & & & & & & & & & & & \\
\hline
\end{tabular}

11

. $\Xi$

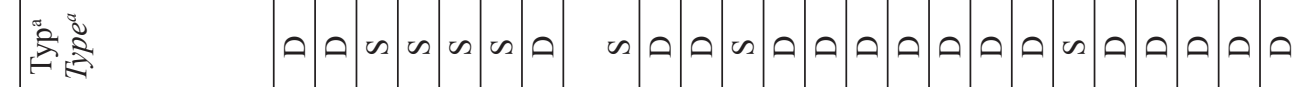
焉

in

:

$\stackrel{\frac{0}{0}}{\frac{0}{0}}$

응

$\frac{1}{0} \stackrel{3}{0}$

흘

ㄴ

泟

$\therefore$

$\forall$

क्ष

\begin{tabular}{|c|c|c|c|c|c|c|c|c|c|c|c|c|c|c|c|c|c|c|c|c|c|c|c|}
\hline \multicolumn{2}{|l|}{ 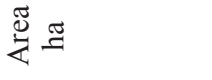 } & $\begin{array}{c}m \\
\infty \\
\infty\end{array}$ & $\left|\begin{array}{l}0 \\
0\end{array}\right|$ & $\begin{array}{l}n \\
? \\
n \\
0\end{array}$ & $\begin{array}{l}0 \\
0 \\
r\end{array}$ & $\begin{array}{l}2 \\
0 \\
n\end{array}$ & 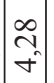 & $\begin{array}{r}\tilde{f} \\
\dot{\sigma}\end{array}$ & $\begin{array}{c}\mathrm{v} \\
\mathrm{i}\end{array}$ & $\exists$ & $\begin{array}{l}= \\
\Rightarrow\end{array}$ & $\tilde{\sigma}$ & & & & $\begin{array}{c}\text { ते } \\
\text { ô }\end{array}$ & $\begin{array}{l}0 \\
\vdots \\
0 \\
0\end{array}$ & $\hat{a}$ & $\left|\begin{array}{l}\infty \\
0 \\
\uparrow \\
\uparrow\end{array}\right|$ & : & 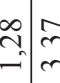 & $\ddot{\circ}$ & $\hat{\sigma}$ \\
\hline \multirow{2}{*}{ 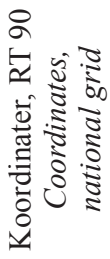 } & $>$ & $\begin{array}{l}\infty \\
\infty \\
\infty \\
0 \\
-1 \\
-\end{array}$ & 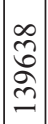 & 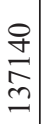 & $\begin{array}{c}\hat{n} \\
\hat{n} \\
\tilde{n} \\
-\end{array}$ & 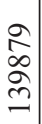 & 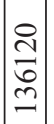 & 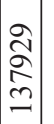 & $\begin{array}{l}m \\
\hat{n} \\
\infty \\
\end{array}$ & $\begin{array}{l}m \\
\vec{\Xi} \\
\stackrel{2}{=}\end{array}$ & $\begin{array}{l}\stackrel{m}{\overrightarrow{ \pm}} \\
\stackrel{2}{n}\end{array}$ & $\begin{array}{l}\infty \\
0 \\
0 \\
0 \\
0 \\
\end{array}$ & & $\widehat{h}$ & 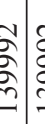 & $\begin{array}{c}\sigma \\
\sigma \\
\sigma \\
\sigma\end{array}$ & 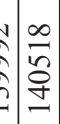 & 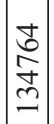 & $\begin{array}{c}m \\
0 \\
\infty \\
-2 \\
=\end{array}$ & 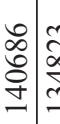 & 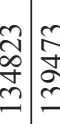 & $\begin{array}{l}\hat{2} \\
\infty \\
\infty \\
0\end{array}$ & 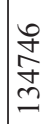 \\
\hline & $x$ & 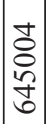 & 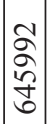 & $\begin{array}{l}8 \\
8 \\
8 \\
\text { ț }\end{array}$ & 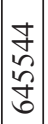 & $\begin{array}{l}\overrightarrow{\hat{\sigma}} \\
\tilde{\tilde{J}} \\
\end{array}$ & $\mid \begin{array}{c}\stackrel{a}{\exists} \\
\stackrel{\mathcal{T}}{\mathcal{J}}\end{array}$ & 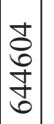 & $\begin{array}{l}\vec{J} \\
\stackrel{f}{f} \\
\text { d }\end{array}$ & $\mid$\begin{tabular}{c}
$\infty$ \\
0 \\
$\infty$ \\
\multirow{2}{*}{} \\
0
\end{tabular} & 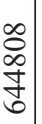 & 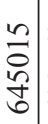 & $\begin{array}{l}\hat{\imath} \\
\hat{\infty} \\
\hat{\delta} \\
\hat{b}\end{array}$ & $\begin{array}{l}5 \\
\vdots \\
5 \\
5\end{array}$ & \begin{tabular}{l|l}
5 \\
$\vdots$ \\
$b$ \\
$b$
\end{tabular} & 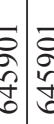 & 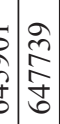 & $\mid \begin{array}{c}- \\
\overrightarrow{1} \\
\infty \\
\widetilde{b}\end{array}$ & 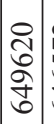 & 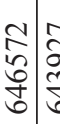 & \begin{tabular}{c|c}
$\widehat{a}$ & \multirow{2}{*}{} \\
$\tilde{b}$ & \multirow{J}{*}{}
\end{tabular} & $\begin{array}{l}0 \\
8 \\
0 \\
\tilde{D} \\
0\end{array}$ & $\begin{array}{l}\infty \\
\infty \\
\infty \\
\hat{\sigma}\end{array}$ \\
\hline 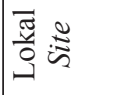 & & -1 & $N$ & $m$ & $\nabla$ & $n$ & 0 & $r$ & $\infty$ & $a$ & 이 & $=$ & $\simeq:$ & -1 & -17 & $\therefore \simeq$ & $D=$ & $\infty$ & 2 & $\stackrel{\overbrace{}}{\bar{c}}$ & $\vec{\sim}$ & $\approx$ & $\stackrel{ \pm}{\sim}$ \\
\hline
\end{tabular}




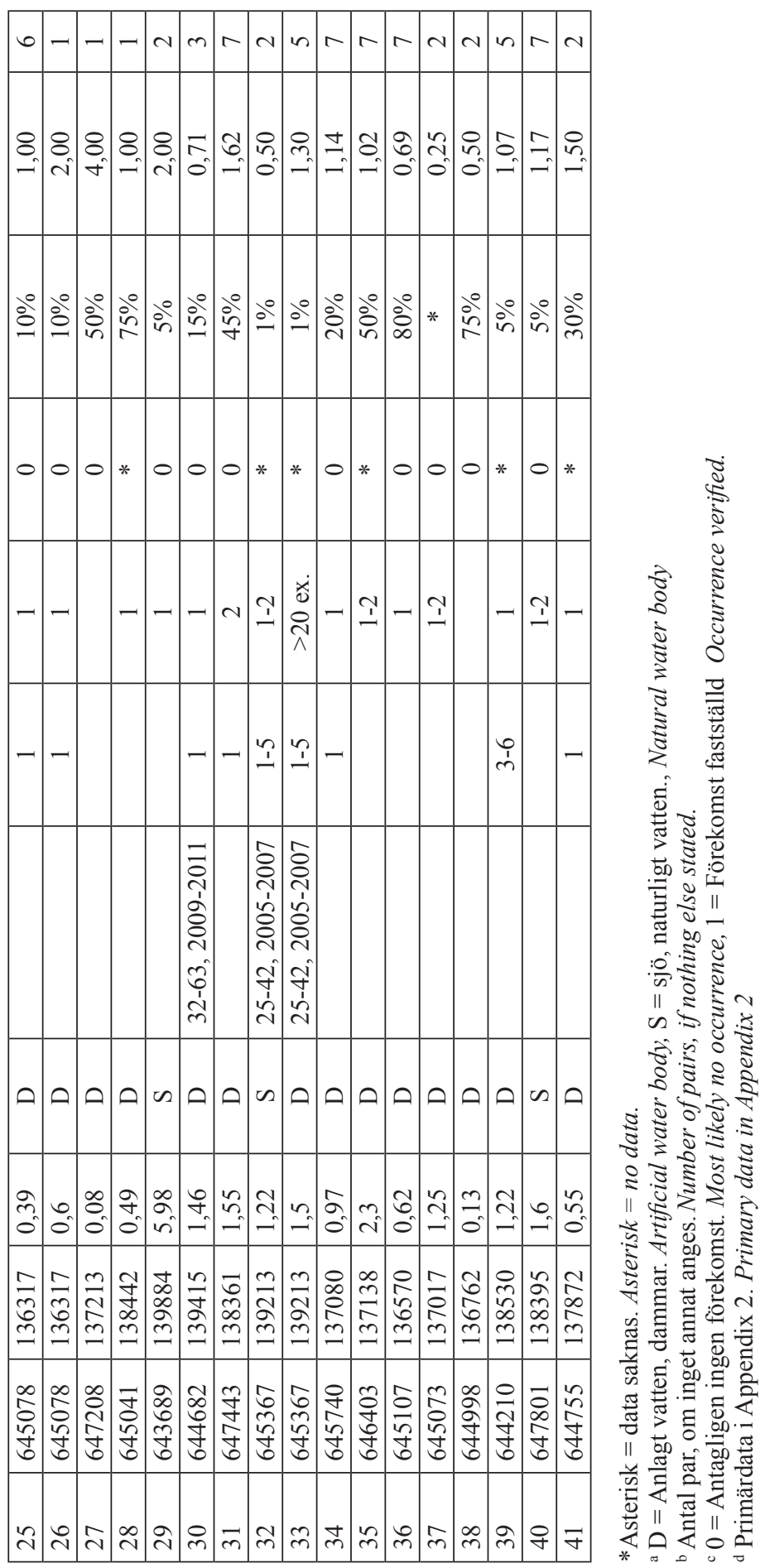




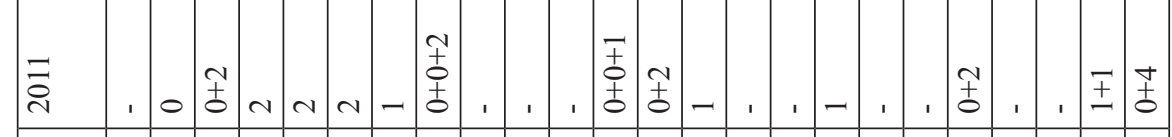

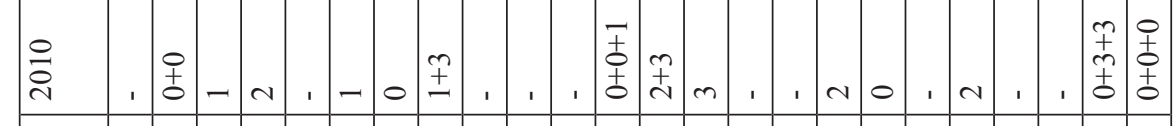

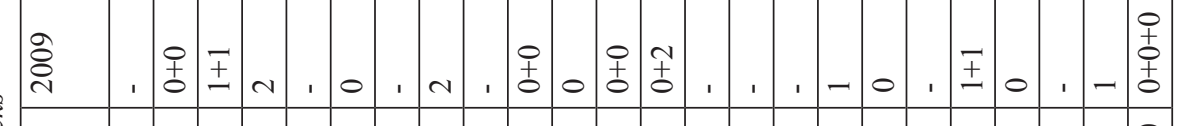

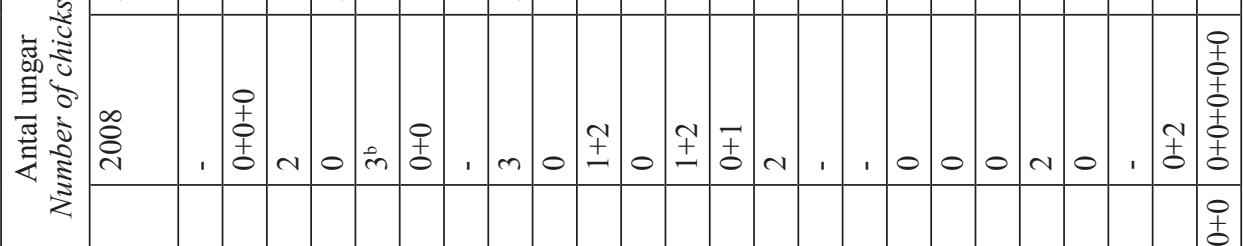

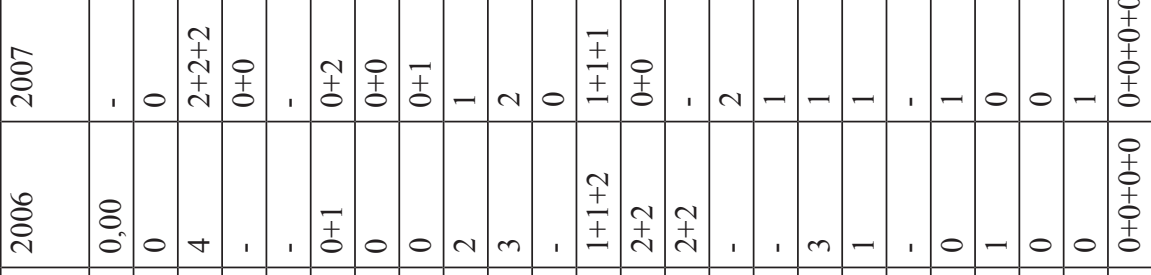

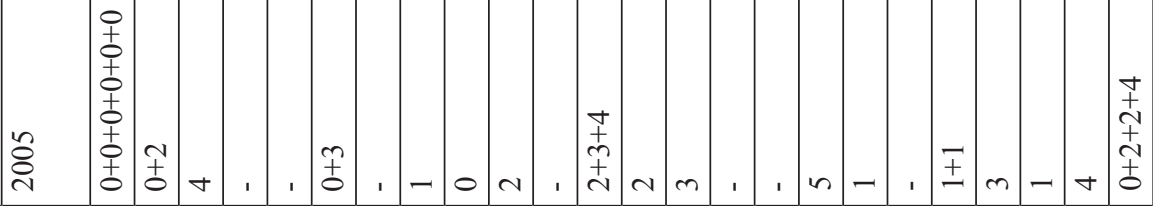

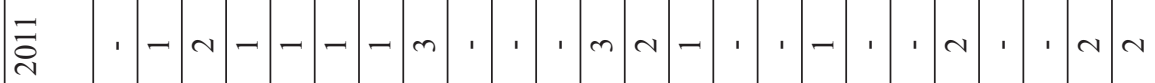

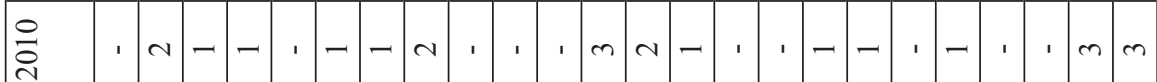

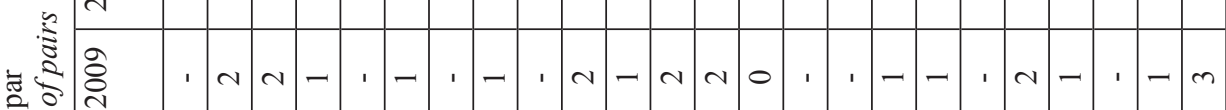

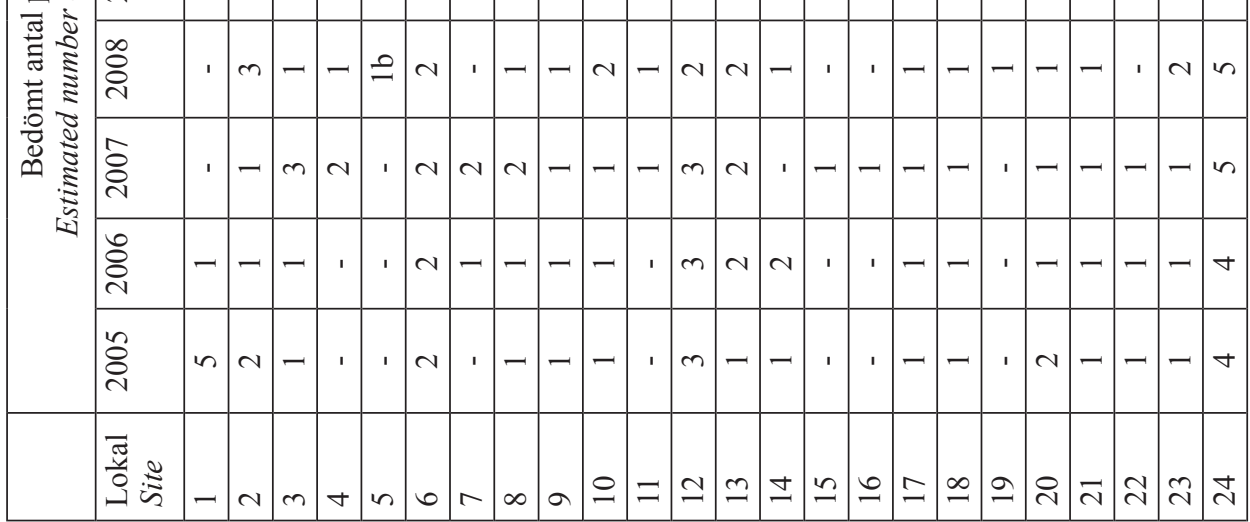




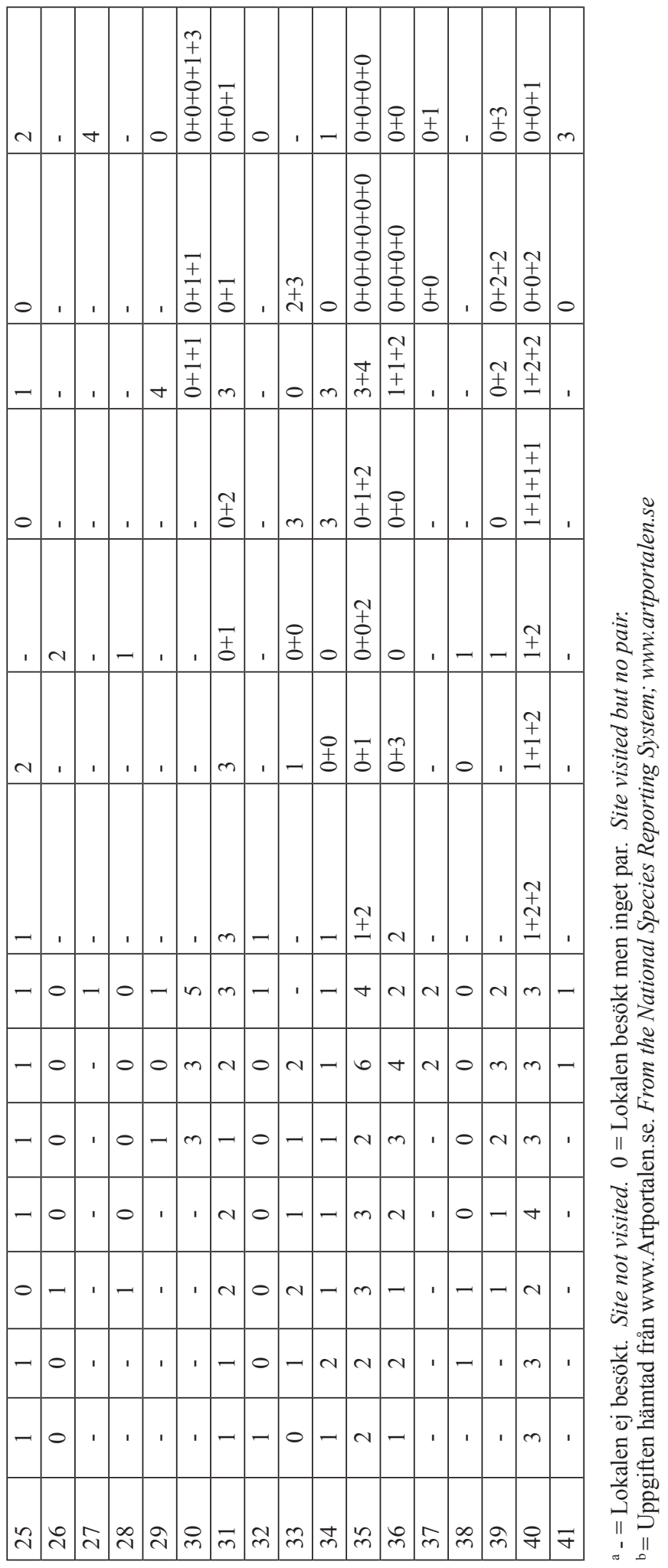

